\section{Thoracoscopic Radiofrequency Ablation Therapy for Hepatocellular Carcinoma Above the Dia- phragm Associated with Intractable Hemothorax}

We have previously described thoracoscopic thermal ablation therapy for hepatocellular carcinoma (HCC) located just beneath the diaphragm, in nine patients with advanced liver cirrhosis [1]. We report here the application of that technique for HCC which had invaded above the diaphragm and was accompanied by intractable hemothorax.

In 1996, a 55-year-old man was treated with interferon for chronic hepatitis caused by hepatitis $\mathrm{C}$ virus (HCV), and his liver function test became normal with negative results for serum HCV-RNA. In 1999, he was diagnosed with HCC on the basis of ultrasonographic examination and an increased level of serum $\alpha$-fetoprotein $(20000 \mathrm{ng} / \mathrm{ml})$. The size of the tumor was $6.8 \mathrm{~cm}$ in $\mathrm{S} 8$ and $3.3 \mathrm{~cm}$ in S2. He was treated with percutaneous radiofrequency ablation therapy [2] after transcatheter arterial emoblization (TAE). In February 2000, he suffered from dyspnea,
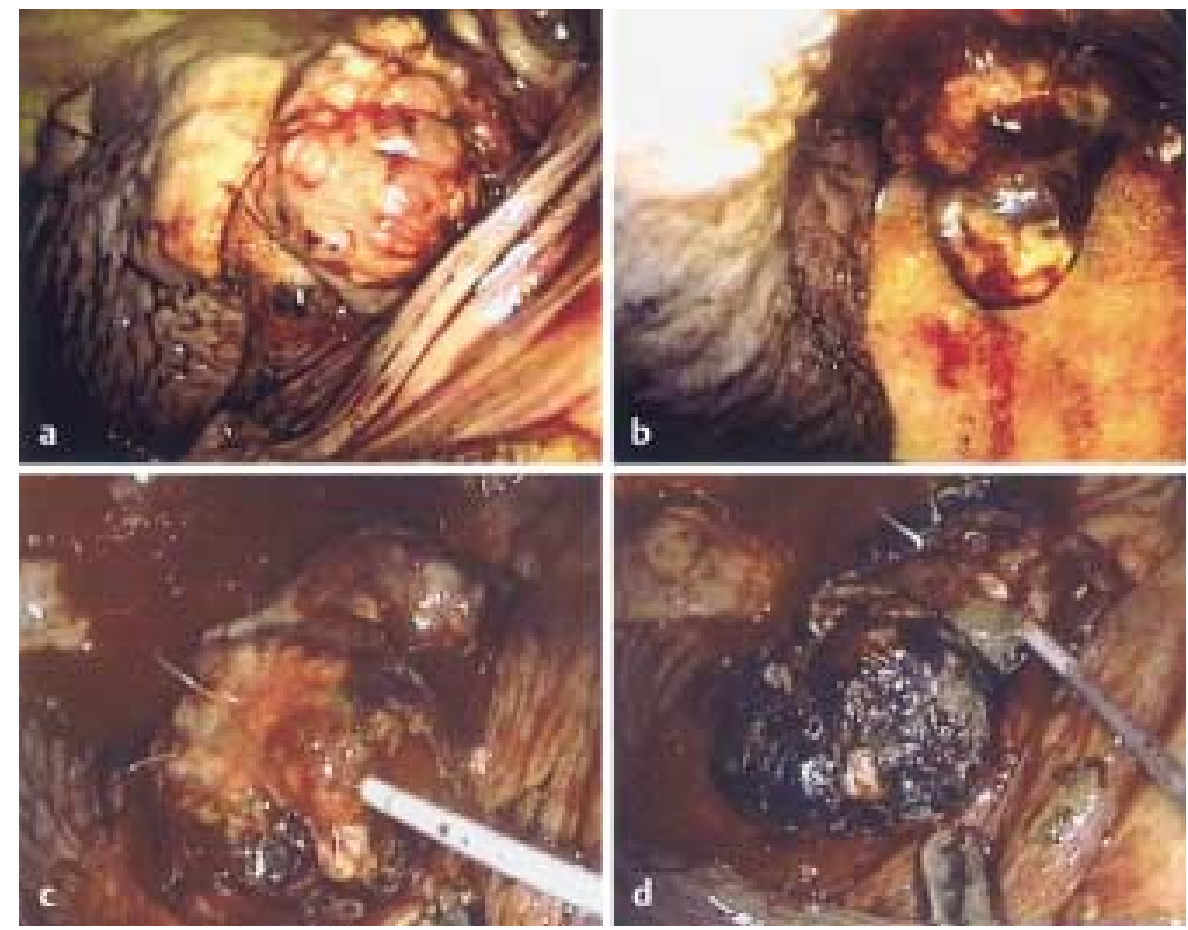

Figure 1 Thoracoscopic views of the hepatocellular carcinoma above the diaphragm. a Anterior tumor before ablation; b posterior tumor with fresh fibrin deposit, before ablation; c during radiofrequency ablation; $\mathbf{d}$ after radiofrequency ablation (the tumor turned black in colour)

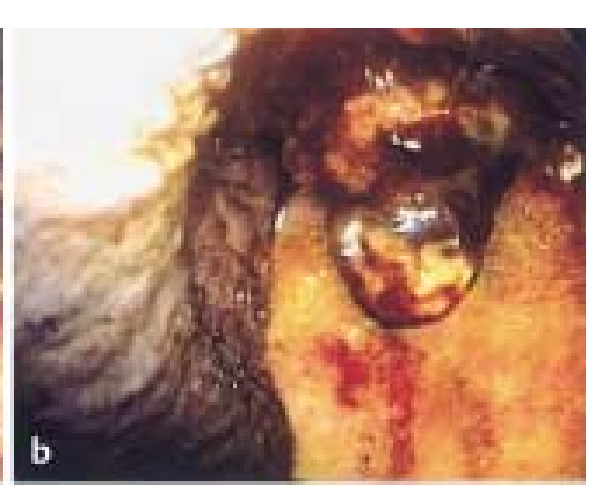

and the chest radiograph showed massive right pleural effusion. Thoracocentesis also showed the bloody effusion. TAE was carried out, but was not effective. Blood transfusion and thoracocentesis were repeated for 2 months. In May 2000, thoracoscopy was carried out under general anesthesia and 21 of bloody pleural effusion were collected. After aspiration, the HCC could be seen invading above the diaphragm (Figures 1a,b). To stop the bleeding, radiofrequency ablation using a LeVeen needle was carried out (Figure 1c), as described previously [1]. Ablation took about 85 minutes in total. After ablation, the tumor turned black in colour (Figure 1 d) and the bleeding was completely stopped. The tumor was too large, and invaded too deeply into the liver, for complete ablation. However, the hemothorax disappeared after the procedure. The patient was discharged 3 days after treatment. There was no complication and no sign of hemothorax after ablation

Corresponding Author

\section{T. Ishikawa, M.D.}

Department of Gastroenterology University of Tokyo

7-3-1, Hongo, Bunkyo-ku

Tokyo, 113-8655

Japan

Fax: $\quad+81-3-5800-9838$

E-mail: takduck-tky@umin.ac.jp 